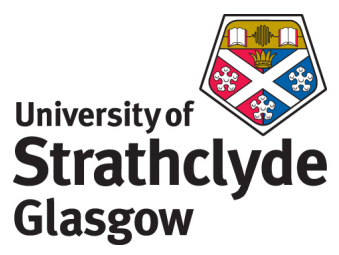

Elgamel, Sherif A.E.H. and Soraghan, J.J. Enhanced monopulse radar tracking using optimum fractional Fourier transform. Radar, Sonar \& Navigation, IET, 4 (6).

http://strathprints.strath.ac.uk/27168/

Strathprints is designed to allow users to access the research output of the University of Strathclyde. Copyright $(C$ and Moral Rights for the papers on this site are retained by the individual authors and/or other copyright owners. You may not engage in further distribution of the material for any profitmaking activities or any commercial gain. You may freely distribute both the url (http://strathprints.strath.ac.uk) and the content of this paper for research or study, educational, or not-for-profit purposes without prior permission or charge. You may freely distribute the url (http://strathprints.strath.ac.uk) of the Strathprints website.

Any correspondence concerning this service should be sent to The Strathprints Administrator: eprints@cis.strath.ac.uk 


\title{
ENHANCED MONOPULSE TRACKING RADAR USING OPTIMUM FRACTIONAL FOURIER TRANSFORM
}

\author{
Sherif A Elgamel and John Soraghan \\ Department of Electronic and Electrical Engineering, University of Strathclyde \\ 204 George Street, Royal Building, G1 1XW, Glasgow, UK \\ phone: +(44)1415482514,e-mail: sherifelgamel73@gmail.com, j.soraghan@eee.strath.ac.uk \\ http:// www.eee.strath.ac.uk
}

\begin{abstract}
Conventional monopulse radar processors are used to track a target that appears in the look direction beam width. The distortion produced when additional targets appear in the look direction beam width can cause severe erroneous outcomes from the monopulse processor. This leads to errors in the target tracking angles that may cause target mistracking. A new signal processing algorithm is presented in this paper which offers a solution to this problem. The technique is based on the use of optimal Fractional Fourier Transform (FrFT) filtering. The relative performance of the new filtering method over traditional based methods is assessed using standard deviation angle estimation error (STDAE) for a range of simulated environments. The proposed system configuration succeeds in significantly cancelling additional target signals appearing in the look direction beam width even if these targets have the same Doppler frequency.
\end{abstract}

Index Term- Interference cancellation, Monopulse radar, Optimum fractional filter

\section{INTRODUCTION}

Monopulse radars are commonly used in target tracking because of their angular accuracy [1]. They provide superior angular accuracy and less sensitivity to fluctuation in the radar cross section (RCS) of 
the target compared to other types of tracking radars [2]. However, these radars are affected by different types of interference which affects the target tracking process and may lead to inaccurate tracking [3-5]. A scenario where more than one target exists in the monopulse radar half power beam width is shown in Fig 1. The resultant distortion due to this interference will affect the induced target error voltage and consequently the radar tracking ability. Seliktar [6] suggested adding more constraints to the monopulse processor to cancel the distortion effect due to more targets appearing in the look direction. However this would require knowledge of the position of the additive targets. In our work we propose the use of an optimal fractional Fourier transform filter to cancel the additional targets' signals that appear in the look direction main beam without adding any more constraints to the monopulse processor.

The paper is organized as follows: Section 2 discusses the structure of a monopulse radar. The mathematical model for the conventional and the spatial adaptive monopulse processors are also described. Section 3 introduces the fractional Fourier transform (FrFT) and explains how the optimum choice of FrFT order is made and also discusses FrFT filtering. Section 4 describes how the standard deviation of angle estimation (STDAE) error is used to evaluate the system performance for monopulse radar processors. Our proposed new structure of the FrFT based monopulse radar processor is discussed in detail in section 5. In section 6 the new algorithm for optimum fractional Fourier transform filtering to reduce the interference due to more than one target in the look direction main beam is derived. A set of simulation results is presented in section 7 for single and multiple targets using the new monopulse processor. Section 8 concludes the paper.

\section{MONOPULSE RADAR PROCESSORS}

A block diagram of a typical monopulse radar is shown in Fig 2. A pulsed chirp signal $c(t)$ is produced from the waveform generator. This is up-converted to the radar carrier frequency, amplified and passed through the duplexer to be transmitted. 


$$
c(t)=\exp \left(j \pi\left(\frac{F_{\text {stop }}-F_{\text {start }}}{T}\right)\left(t-\frac{T}{2}\right)^{2}\right)
$$

where $t$ is the time, $T$ is the chirp time duration (pulse duration), $F_{\text {start }}$ is the chirp start frequency, and $F_{\text {stop }}$ is the chirp stop frequency.

The down-converted received signal passes through a band limited Gaussian filter before passing through the chirp matched filter to maximize the target return signal. The target information parameters (azimuth angle, elevation angle, and target range) are then calculated by the monopulse processor from the filtered signal. A typical monopulse processor for phased array radars is obtained by appropriately phasing the individual array channels to obtain sum and difference outputs $[4,7]$. The ratio of the difference-to-sum outputs provides the measure by which the angle offset from the beam axis (i.e. look direction) is determined. The updated angle measurement is used to realign the beam axis with the target. The structure of monopulse radar shown in Fig 2 is repeated $N$ times ( $N$ equal to the of array antenna elements). Thus each antenna will have its own complete receiving system and all the output data will be processed in only one monopulse processor.

\subsection{Conventional Monopulse Processor}

The conventional monopulse radar processor is a non adaptive configuration. This processor consists of two sets of weights set to the sum and difference steering vectors, respectively $[8,9]$ :

$$
w_{\Sigma}=a\left(v_{l}\right), \quad w_{\Delta}=\left.\frac{\partial a(v)}{\partial v}\right|_{v_{l}}
$$

where $a(v)=\frac{e^{-j \pi v(n-1)}}{\sqrt{N}}\left[\begin{array}{llll}1 & e^{j 2 \pi v} & \ldots \ldots \ldots & e^{j 2 \pi v(N-1)}\end{array}\right]$ is the centre phase normalized steering vector in the look direction, $N$ is the number of antenna, $v$ is the spatial steering frequency. The sum and difference outputs are given in terms of the respective processors, 


$$
z_{\Sigma}(l)=w_{\Sigma} \mathbf{x}(l), \quad z_{\Delta}(l)=w_{\Delta} \mathbf{x}(l)
$$

where $\mathbf{x}(l)$ is the $N \times 1$ spatial snapshot at time instant $l$. The real part of the ratio of difference to sum outputs is known as the error voltage defined as $[6,8]$

$$
\varepsilon_{v}(l)=\mathfrak{R}\left\{\frac{z_{\Delta}(l)}{z_{\Sigma}(l)}\right\}
$$

This error voltage conveys purely directional information that must be converted to an angular form via a mapping function. The mapping function, called the monopulse response curve (MRC) [8, 9], is given in terms of the ratio of the two receive beam pattern functions. It represents the ideal response of the antenna to targets across various angles, so $\mathrm{MRC}, M(\phi)$ is defined as

$$
M(\phi)=\Re\left\{\frac{W_{\Delta}(\phi)}{W_{\Sigma}(\phi)}\right\}
$$

where $W_{\Delta}(\phi)$ and $W_{\Sigma}(\phi)$ are the monopulse beam pattern for the sum processor and the difference processor respectively at look direction angle $\phi$. So MRC is the ratio of difference to sum beam patterns and represented the ideal error voltage response to targets arriving from a particular angular region about boresight.

\subsection{Spatial Adaptive Monopulse Processor}

The spatial processor is an adaptive configuration. Adaptive sum and difference beams are formed by applying sum and difference unity gain constraints in the look direction

$$
w_{\Sigma} v_{\Sigma}=1, \quad w_{\Delta} v_{\Delta}=1
$$

where $v_{\Sigma}=a\left(v_{l}\right)$ and $v_{\Delta}=\left.\frac{\partial a(v)}{\partial v}\right|_{v_{l}}$. To minimize the mean square error output from the processor, the sum and difference weights may be written in the following form [6, 10-12]:

$$
w_{\Sigma}=\frac{R_{x}^{-1} v_{\Sigma}}{v_{\Sigma}^{H} R_{x}^{-1} v_{\Sigma}}, \quad w_{\Delta}=\frac{R_{x}^{-1} v_{\Delta}}{v_{\Delta}^{H} R_{x}^{-1} v_{\Delta}}
$$


where $R_{x}$ is the covariance matrix of the input data [13]to the processor with diagonal loading [14, 15] . In the construction of $R_{x}$ tries to exclude as much as possible the target data from the input data to the processor (target range bin interval are deleted from the processed data).

\section{FRACTIONAL FOURIER TRANSFORM (FrFT)}

The FrFT is the generalized formula for the Fourier transform that transforms a function into an intermediate domain between time and frequency $[16,17]$. The signals with significant overlap in both the time and frequency domain may have little or no overlap in the fractional Fourier domain. As illustrated in Fig 3, signals $S_{1}$ and $S_{2}$ can be separated in the FrFT domain using an order $a$.

The fractional Fourier transform of an arbitrary function $x(t)$, with an angle $\alpha$, is defined as:

$$
X_{\alpha}\left(t_{a}\right)=\int_{-\infty}^{\infty} x(t) K_{\alpha}\left(t, t_{a}\right) d t
$$

where $K_{\alpha}\left(t, t_{a}\right)$ is the transformation Kernel, $t_{a}$ is the transformation of $t$ to the $a^{\text {th }}$ order, and $\alpha=a \pi / 2$ with $a \in \mathfrak{R} . K_{\alpha}\left(t, t_{a}\right)$ is calculated from:

$$
K_{\alpha}\left(t, t_{a}\right)= \begin{cases}\sqrt{\frac{1-j \cot \alpha}{2 \pi}} e^{j \frac{t^{2}+t_{a}{ }^{2}}{2} \cot \alpha-j t_{a} t \csc \alpha} & \text { if } \alpha \text { is not a multiple of } \pi \\ \delta\left(t-t_{a}\right) & \text { if } \alpha \text { is a multiple of } 2 \pi \\ \delta\left(t+t_{a}\right) & \text { if } \alpha+\pi \text { is a muliple of } 2 \pi\end{cases}
$$

\subsection{Optimum FrFT domain}

The optimum value for $a$ for a chirp signal may be written as [18]:

$$
a_{o p t}=-\frac{2}{\pi} \tan ^{-1}\left(\frac{\delta f}{2 \gamma \times \delta t}\right)
$$


where $\delta f$ is the frequency resolution $\left(\delta f=F_{s} / N\right), \delta t$ is the time resolution $\left(\delta t=1 / F_{s}\right), F_{s}$ is the sampling frequency, and $\gamma$ is the chirp rate parameter. Eq 10 can be used to either calculate the optimum FrFT order or to estimate the chirp rate of a signal for a given FrFT order.

\subsection{Optimum FrFT Filter}

A signal observation model $z$ may be described by:

$$
z=\boldsymbol{H}+\boldsymbol{y}
$$

where $\boldsymbol{x}$ is the system useful signal, $\boldsymbol{y}$ is the sum of all distortion signals, and $\boldsymbol{H}$ is the matrix characterizing the degradation process. The cross correlation matrix $R_{x_{a} z_{a}}$ is the cross correlation between $\boldsymbol{x}$ and $z$ in the FrFT domain of order $a$. The auto correlation matrix $R_{z_{a} z_{a}}$ is the auto correlation for signal $z$ in the FrFT domain of order $a$. These matrices may be calculated from the following equations [19]

$$
\begin{gathered}
R_{x_{a} z_{a}}=F^{a} R_{x x} \boldsymbol{H}^{H} F^{-a} \\
R_{z_{a} z_{a}}=F^{a}\left(\boldsymbol{H} R_{x x} \boldsymbol{H}^{H}+R_{y y}\right) F^{-a}
\end{gathered}
$$

where $F^{a}$ and $F^{-a}$ are discrete FrFT matrices of order $a$ and $-a$, respectively, $R_{x x}$ and $R_{y y}$ are the covariance matrix for the signals $\boldsymbol{x}$ and $\boldsymbol{y}$ respectively, and $H$ is the Hermitian transpose.

The optimum filter $g_{o p t, j}$ to filter the $\boldsymbol{y}$ signal in the FrFT domain is given by:

$$
g_{\text {opt }, j}=\frac{R_{x_{a} z_{a}}(j, j)}{R_{z_{a} z_{a}}(j, j)} \quad j=1,2, \ldots \ldots, m
$$

where $m$ is the signal length.

The filtered signal $\boldsymbol{x}^{\prime}$ in the time domain is calculated from [16, 20]

$$
\boldsymbol{x}^{\prime}=F^{-a} \Lambda_{g} F^{a} z
$$

where $\Lambda_{g}$ is a diagonal matrix whose diagonal consists of the elements of the vector $g_{o p t, j}$. 


\section{PERFORMANCE MEASURE FOR MONOPULSE RADAR}

One commonly used measure for the performance of monopulse radar processors is the standard deviation of the angle error (STDAE). The STDAE is determined with a target injected randomly across range and angle within the main beam and the corresponding angle error averaged over range [6]

$$
\sigma_{\varepsilon_{\phi}}=\sqrt{E\left\{\left|\varepsilon_{\phi}\right|^{2}\right\}}
$$

where $\varepsilon_{\phi}=\hat{\phi}-\phi, \phi$ is the target angle, and $\hat{\phi}$ is measured angle. When there is only one target signal without any distortion signal (other targets in the look direction, jamming signal, clutter, thermal noise, and terrain scattering interference), both $\phi$ and $\hat{\phi}$ are nearly equal, hence the STDAE is near zero and its value will increase due to the existence of the distortion signal.

\section{A NEW STRUCTURE OF MONOPULSE RADAR}

The proposed new structure of the monopulse radar is shown in Fig 4. In the reception mode as previously described, the received signal $s(t)$ in the baseband passes through a band pass Gaussian filter and can be written in the following form:

$$
s(t)= \begin{cases}{\left[\left(A e^{-j 2 \pi \phi_{o}} e^{\left.j \pi\left(\frac{\left.F_{\text {stop }}-F_{\text {star }}\right)\left(t-T_{\text {start }} \frac{T}{2}\right)^{2}}{T}\right) \cdot F_{d}\right] \times F_{\phi}}\right.\right.} & T_{\text {start }}<t<T_{\text {start }}+T \\ 0 & \text { elsewhere }\end{cases}
$$

where $A$ is the received signal amplitude, $\phi_{o}$ is a random phase shift, and $T_{\text {start }}$ is the start time of the returned pulse. The start time $T_{\text {start }}$ depends on the target range $R_{t}$ and is determined from:

$$
T_{\text {start }}=\frac{2 \times R_{t}}{c}
$$

where $c$ is the speed of light with approximate value $3 \times 10^{8}$. The Doppler shift and delay effect on the target chirp signal is determined by the dot product of the chirp signal by the Doppler and delay vector $F_{d}$ 


$$
F_{d}=\exp \left(j 2 \pi f_{d}\left(t-T_{\text {start }}\right)\right)
$$

where $f_{d}$ is the target Doppler frequency.

For the uniform line phased array receiving antenna, the antenna phase factor $F_{\phi}$ is introduced by

$$
F_{\phi}=\exp \left(-j 2 \pi f_{c}\left(T_{\text {start }}-N \times \Delta t\right)\right)
$$

where $N$ is a vector represented as $0: N-1$, and $\Delta t$ is calculated from

$$
\Delta t=\frac{D \times \sin \phi_{t}}{c}
$$

where $D$ is the separation between the antenna elements, $\phi_{t}$ is the target angle from the antenna boresight.

As seen in Fig 4 the optimum fractional filter obtains information about the shape of the chirp signal from the waveform generator and the updated target range from the range calculation. This information is used to determine the optimal FrFT domain. This will be described in detail in section 6 .

The new monopulse radar processor illustrated in Fig 5 consists of $N$ receiving channel in which the received signal from each of the $N$ antenna elements will fill $L$ range gates. The total radar data size is therefore equal to $N \times L$ for each pulse return. The optimum FrFT domain is calculated for each receiving channel data with size $1 \times L$ to filter the signal in the fractional domain. The resultant filtered data (useful signal) is converted back from the optimum FrFT domain using inverse FrFT processor to the time domain. The $1 \times L$ data output from $N$ FrFT processors are applied to azimuth, elevation, and range calculator to determine the target information parameters.

\section{OPTIMAL FrFT FOR MONOPULSE RADAR}

The following steps describe the proposed algorithm that may be employed to cancel more than one target signal arriving in the look direction of the main beam while extracting the $1^{\text {st }}$ target signal:

1. Determine the optimal fractional domain for the $1^{\text {st }}$ target signal.

2. Calculate the correlation matrix for the $1^{\text {st }}$ target and the other targets. 
3. Calculate the cross correlation matrix for the $1^{\text {st }}$ and the other targets in the optimum FrFT domain and the auto correlation matrix of the additive targets in the optimum FrFT domain.

4. Design the optimum filter in the fractional domain.

5. Extract the useful signal (the $1^{\text {st }}$ target signal) by using the optimum fractional transform matrix.

6. Transform the useful signal to time domain by using the inverse optimum fractional transform matrix

The mathematical description for the previous steps is now described in detail:

Applying the signal model of Eq 11 to our radar system in which $\boldsymbol{H}$ is considered to be unity matrix (no system degradation)

$$
z=x+y
$$

where the useful signal $\boldsymbol{x}$ is the $1^{\text {st }}$ target signal and the distortion signal $\boldsymbol{y}$ is the sum of the additional targets' signals (in the simulation, the 2nd target signal and the 3rd target signal).

The target received signal is a chirp signal given by Eq 17. The optimum FrFT order $a_{\text {opt }}$ for this chirp can be computed by applying Eq 10 to the radar system as

$$
a_{\text {opt }}=-\frac{2}{\pi} \tan ^{-1}\left(\frac{F_{s}^{2} \times T}{\left(F_{\text {stop }}-F_{\text {start }}\right) \times L}\right)
$$

The required information to calculate correlation matrices is obtained from the fact that we have previous knowledge of the target position (already tracked before the other targets enter the radar look direction) and from the sample signal of the waveform generator (parameters of the transmitted chirp signal). So $R_{x x}$ apart from a scale factor $A$ is computed as:

$$
R_{x x}=E\left(x \cdot x^{H}\right)
$$

where $x$ is the chirp signal of the $1^{\text {st }}$ target at range $R_{t}$ :

$$
\boldsymbol{x}=e^{-2 j \pi \times \phi_{x}} e^{\left(j \pi\left(\frac{F_{\text {stop }}-F_{\text {start }}}{T}\right)\left(t_{n}-T_{\text {start }}-\frac{T}{2}\right)^{2}\right)}
$$


where $\phi_{x}$ is a random phase shift similar to that used in Eq 17 and $T_{\text {start }}$ is calculated from Eq 18 for target at range $R_{t}$.

In the same fashion $R_{y y}$ is calculated from:

$$
R_{y y}=E\left(y \cdot y^{H}\right)
$$

where $\boldsymbol{y}$ is the chirp signal at the other targets range:

$$
\boldsymbol{y}=e^{-2 j \pi \times \phi_{y}} e^{\left(j \pi\left(\frac{F_{\text {stop }}-F_{\text {satr }}}{T}\right)\left(t_{n}-T_{\text {start }}-\frac{T}{2}\right)^{2}\right)}
$$

where $\phi_{y}$ is a random phase shift similar to that used in Eq 17 and $T_{\text {start }}$ is calculated from Eq 18 for target at range $R_{t}+\Delta R_{t}$ and $\Delta R_{t}$ is the maximum range difference between the $1^{\text {st }}$ target and any of the additive targets that cannot be resolved by a range gate canceller. $\Delta R_{t}$ can also be considered as the number of range bin occupied by the $1^{\text {st }}$ target. $\Delta R_{t}$ in Eq 27 ensures that no need to acquire any information about the range of the additive targets.

The next step is to calculate the cross correlation matrix $R_{x_{a} z_{a}}$ for the $1^{\text {st }}$ and the other targets and the auto correlation matrix $R_{z_{a} z_{a}}$ of the other targets in the calculated optimum FrFT domain by applying Eq 12 and Eq 13 respectively as

$$
\begin{gathered}
R_{x_{a} z_{a}}=F^{a_{o p t}} R_{x x} I^{H} F^{-a_{o p t}} \\
R_{z_{a} z_{a}}=F^{a_{o p t}}\left(I R_{x x} I^{H}+R_{y y}\right) F^{-a_{o p t}}
\end{gathered}
$$

Then the optimum filter in the optimum FrFT domain $g_{\text {opt }, j}$ is given by

$$
g_{\text {opt }, j}=\frac{R_{x_{a} z_{a}}(j, j)}{R_{z_{a} z_{a}}(j, j)} \quad j=1,2, \ldots \ldots, L
$$

The filtered signal $\boldsymbol{x}^{\prime}$ in the time domain is calculated from Eq 15. All the outputs signals from the $N$ FrFT filters are supplied to the monopulse processor (the processors mathematical models were presented in section 2) to calculate the target information. 


\section{SIMULATION RESULTS}

Initially we will simulate one target for the conventional and spatial adaptive monopulse processors. Then we will consider the case with multiple targets in the radar look direction main beam and examine the subsequent degradation on the system performance as measured by STDAE curves. The simulation of two targets for the two monopulse processors using optimum FrFT filter will be presented and the enhancement in the target tracking will be presented.

In the simulations the radar comprises an array of 14 elements spaced 1/3 meters apart. The radar pulse width is 100 microseconds and the pulse repetition interval of 1.6 milliseconds for a $435 \mathrm{MHz}$ carrier. A $200 \mathrm{kHz}$ Gaussian band pass filters exists at the front end of each $N$ receiver to filter the incoming data returns prior to sampling. The incoming baseband signals are sampled at $1 \mathrm{MHz}$. Also it is assumed that the radar operating range is 100:200 range bins with a starting window at 865 microseconds and a window duration of 403 microseconds. The target is considered at range bin=150 at angle $32^{\circ}$ from the look direction with target signal to noise ratio (SNR) set to $50 \mathrm{~dB}$ with Doppler frequency $150 \mathrm{~Hz}$.

\subsection{Single Target}

Using Eq 2 it can be seen in Fig 6(a) that the sum pattern has maximum at the look direction angle $32^{\circ}$ and null at the same angle for the difference pattern in Fig 6(b). A well sloped curved for monopulse error voltage (the ratio of the derivative of the difference pattern over the sum) is calculated from Eq 4 and is shown in Fig 6(c). It determines the target position by mapping this voltage onto the monopulse response

curve (MRC) to get $\hat{\phi}$. Any distortion to this curve will affect the target position calculation. The processor output is determined from Eq 3. From Fig 6(d), it is seen that there is only one target at the range bin 150 in the operating radar range bins.

The spatial processor pattern is calculated from $\mathrm{Eq} 7$, the processor output from $\mathrm{Eq} 3$, and the monopulse error voltage from Eq 4. It is shown in Fig 7(a) that the sum pattern has maximum at the look direction 
angle $32^{\circ}$ and null at the same angle for the difference pattern in Fig 7(b). It looks like the same pattern shape of the conventional processor because there is no signal interference due which the sum and difference patterns change their shape to try to cancel the effect of this interference. Again a well sloped curved for monopulse error voltage is obtained. From Fig 7(d), it is seen that there is only one target at the range bin 150 in the operating radar range bins.

\subsection{Multiple Targets}

In the simulation for two targets, we consider that the second target's SNR equals $53 \mathrm{~dB}$ (double the power of the $1^{\text {st }}$ target), at an angle that varies randomly near to the $1^{\text {st }}$ target (the radar is locked on the $1^{\text {st }}$ target before the additive targets enter the radar half power beam width) but still in the look direction beam width), and at range bin 153 . Note that this second target cannot be resolved because the $1^{\text {st }}$ target occupied bins include 7 bins with the same Doppler frequency of the $1^{\text {st }}$ target as seen in Fig 6 and Fig 7. For the three targets scenario, we consider that the third target SNR equal $50 \mathrm{~dB}$ (equal power of $1^{\text {st }}$ target), also at an angle that varies randomly near to the $1^{\text {st }}$ target but still in the look direction beam width), and at range bin 147 (nearer than the 1st target to the tracking radar) with the same Doppler frequency as the $1^{\text {st }}$ target.

\subsubsection{Two Targets Scenario}

The conventional and the spatial processor outputs using Eq 3 are seen in Fig 8(b) and Fig 9(b) respectively. It is clear that in these figures that the second target cannot be cancelled using range gate canceller (overlapped with the $1^{\text {st }}$ target). The two target problem causes deviation in the monopulse error voltages from their original values to distorted curves as seen in Fig 8(a), and Fig 9(a). This distortion in the error voltage will affect the tracking angle of the $1^{\text {st }}$ target resulting in a probable mistracking outcome. 
From Fig 8(d), the STDAE for the conventional processor is much higher at 2.9 for different target SNR (from 20-100 dB), so the system is completely distorted and the radar cannot track the $1^{\text {st }}$ target. In the case of the spatial adaptive processor in Fig 9(d), it starts to achieve good tracking results from approximately $60 \mathrm{~dB}$ because of the adaptive characterization of the beam pattern that attempts to cancel the $2^{\text {nd }}$ target signal. Despite the low STDAE values (average value 0.3 ) the processor still introduces considerable error in the $1^{\text {st }}$ target angle calculation.

\subsubsection{Three Targets Scenario}

The conventional and the spatial processor outputs using Eq 3 are seen in Fig 8(b) and Fig 9(b) respectively. It can be seen in these figures that both the additive targets cannot be cancelled using range gate canceller (overlapped with the $1^{\text {st }}$ target). There is now significant deviation in the monopulse error voltages from their original values to distorted curves as seen in Fig 8(a), and Fig 9(a) due to the third target. From Fig 8(d), the STDAE for the conventional processor is much higher at 2.9 for different target SNR, so the system is completely distorted and the radar cannot track the $1^{\text {st }}$ target. In case of the spatial adaptive processor in Fig 9 (d), it starts to achieve good tracking result from approximately $70 \mathrm{~dB}$.

\subsection{Monopulse Processors using Optimum FrFT Filter}

Substituting the specific monopulse radar parameters in Eq 23, the order of the optimal FrFT domain $a_{\text {opt }}$ is computed as 1.7074 . Following the steps described in section 6 , we calculate the correlation matrix for the $1^{\text {st }}$ target $R_{x x}$ and the additive targets $R_{y y}$ by considering $\Delta R_{t}=7$ range bin (more than 7 range bin there is no problem because the radar can cancel the additive targets using rage gate canceller) by using Eq 24 and Eq 26 respectively. All the steps in section 6 are continued until the filtered data is produced.

\subsubsection{Two Targets Scenario with FrFT}

Applying the filtered data to the radar processors to calculate the processors outputs using Eq 3, it is seen from Fig 8(c) and Fig 9(c) that only one strong target appears in the output and the $2^{\text {nd }}$ target is significantly suppressed (more than $20 \mathrm{~dB}$ reduction). As seen in Fig 8(a) and Fig 9(a), the resulting 
monopulse curve for the two targets scenario with FrFT are nearly identical to their original values (only one target). As a result the significance of the distortion due to the $2^{\text {nd }}$ target in the monopulse look direction has been minimised. The resultant STDAE using Eq 16 for different SNR (20:100 dB) for the conventional processor is particularly low (average value less than 0.1) as shown in Fig 8(d). In Fig 9(d) the STDAE for the spatial processor in case of two targets using FrFT are particularly low (average value less than 0.1). The new system configuration will enhance the system performance for the two target scenario at all SNR for the considered radar processors. If the scenario has only targets in the tracked target background then the calculated STDAE will decrease to average 0.1 due to the highly signal suppression in this case. This implies that both processors are able to track the first target correctly and the introduced error due to the existence of the additive target is significantly reduced.

\subsubsection{Three Targets Scenario with FrFT}

Applying the filtered data to the radar processors to calculate the processors outputs using Eq 3, it is seen from Fig 8(c) and Fig 9(c) that only one strong target appears in the output and the $2^{\text {nd }}$ target is significantly suppressed (more than $20 \mathrm{~dB}$ reduction) and the $3^{\text {rd }}$ target is suppressed (more than $5 \mathrm{~dB}$ reduction). The same results are obtained when only one additive targets is exists, in other words when only one additive target exists, its signal is suppressed by $5 \mathrm{~dB}$ reduction and $20 \mathrm{~dB}$ reduction for the near target and far target respectively. As seen in Fig 8(a) and Fig 9(a), the resulting monopulse curve for the three targets scenario with FrFT are slightly different to their original values (only one target). As a result the problem of the distortion due to the 3rd target in the monopulse look direction has been resolved. The resultant STDAE using Eq 16 for different SNR (20:100 dB) for the conventional processor is low (average value less than 0.3) as shown in Fig 8(d). In Fig 9(d) the STDAE for the spatial processor in case of three targets using FrFT are particularly low (average value less than 0.3). The higher values for STDAE in this case because the 3rd target is nearer to the radar than the 1st one. In other words if the 3rd target is in the background of the 1st target, the STDAE will reduce to 0.1 (similar to the two target scenario). 
The previous suppression of the additive targets signals power and also the enhancements in the STDAE values were tested for up to six targets (3 near target and 3 far targets) and generally a similar SNR reduction of approximately $20 \mathrm{~dB}$ will be observed for all far targets while a SNR reduction of approximately $5 \mathrm{~dB}$ was observed for all near targets.

\section{CONCLUSION}

In this paper we have presented a new solution for the distortion problem due to the unwanted targets appearing in the monopulse look direction main beam. The proposed system configuration with the optimum $N$ FrFT filters succeeds to effectively reduce the additive targets' signal and minimize the STDAE for the both considered monopulse processors. A very high improvement in the radar tracking ability for different SNR (because of very low STDAE) is gained by using the suggested cancelling technique (more than $20 \mathrm{~dB}$ reduction for the far targets and more than $5 \mathrm{~dB}$ reduction for the near targets). One of the key advantages of the proposed system is that it works in an excellent manner when only one target in the look direction (normal case) as well as when more than one target exists in the look direction. In our future work, we will consider that in addition to the additive targets appear in the radar look direction, a jamming signal will also interfere through the radar main lobe and side lobes.

\section{ACKNOWLEDGMENT}

The authors would like to thank the anonymous respective reviewers for their valuable comments.

\section{REFRENCES}

[1] R. Klemm and U. Nickel, "Adaptive monopluse with STAP," in Radar, 2006. CIE '06. International Conference on, 2006, pp. 1-4.

[2] M. I. Skolnik, Radar Handbook-Second edition: McGraw-Hill, Inc., 1990.

[3] S. A. Elgamel and A. H. Makaryous, "Performance of Modern Guided Systems in Presence of Jamming Signal" in 9th international conference on aerospace science and aviation technology Military Technical College, Cairo, Egypt, 2001. 
[4] A. D. Seifer, "Monopulse-radar angle tracking in noise or noise jamming," Aerospace and Electronic Systems, IEEE Transactions on, vol. 28, pp. 622-638, 1992.

[5] S. A. Elgamel and J. J. Soraghan, "Target Tracking Enhancement Using A Kalman Filter In The Presence Of Interference," in Geoscience and Remote Sensing Symposium, 2009. IGARSS 2009. IEEE International, Cape Town, South Africa, 2009.

[6] Y. Seliktar, "Space- Time Adaptive Monopulse Processing." vol. PhD: Georgia Institute of Technology, 1998.

[7] H. Meikle, Modern Radar Systems Artech House, Inc., 2008.

[8] S. Yaron, B. W. Douglas, and E. J. Holder, "A space/fast-time adaptive monopulse technique." vol. 2006: Hindawi Publishing Corp., pp. 218-218.

[9] Y. Seliktar, D. B. Williams, and E. J. Holder, "Adaptive monopulse processing of monostatic clutter and coherent interference in the presence of mainbeam jamming," in Signals, Systems \& Computers, 1998. Conference Record of the Thirty-Second Asilomar Conference on, 1998, pp. 1517-1521 vol.2.

[10] A. Papoulis, Probability, Random Variables, and Stochastic Processes, third edition ed.: McGrawHill Inc., NY, NY, 1991.

[11] D. H. Johnson and D. E. Dudgeon, Array Signal Processing: Prentice Hall, Englewood Cliffs, NJ, 1993.

[12] R. C. Davis, L. E. Brennan, and L. S. Reed, "Angle Estimation with Adaptive Arrays in External Noise Fields," Aerospace and Electronic Systems, IEEE Transactions on, vol. AES-12, pp. 179$186,1976$.

[13] I. S. Reed, J. D. Mallett, and L. E. Brennan, "Rapid Convergence Rate in Adaptive Arrays," Aerospace and Electronic Systems, IEEE Transactions on, vol. AES-10, pp. 853-863, 1974.

[14] S. M. Kogon, D. B. Williams, and E. J. Holder, "Beamspace techniques for hot clutter cancellation," in Acoustics, Speech, and Signal Processing, 1996. ICASSP-96. Conference Proceedings., 1996 IEEE International Conference on, 1996, pp. 1177-1180 vol. 2.

[15] M. K. Stephen, "adaptive Array Processing Techniques for Terrain Scattered Interference Mitigation." vol. PhD: Georgia Institute of Technology, 1996.

[16] A. Kutay, H. M. Ozaktas, O. Ankan, and L. Onural, "Optimal filtering in fractional Fourier domains," Signal Processing, IEEE Transactions on, vol. 45, pp. 1129-1143, 1997.

[17] M. A. Kutay, H. M. Ozaktas, L. Onural, and O. Arikan, "Optimal filtering in fractional Fourier domains," in Acoustics, Speech, and Signal Processing, 1995. ICASSP-95., 1995 International Conference on, 1995, pp. 937-940 vol.2.

[18] C. Capus and K. Brown, "Short-Time fractional fourier methods for the time-frequency representation of chirp signals," The Journal of the Acoustical Society of America, vol. 113(6), pp. 3253-63, 2003.

[19] C. Candan, M. A. Kutay, and H. M. Ozaktas, "The discrete fractional Fourier transform," Signal Processing, IEEE Transactions on, vol. 48, pp. 1329-1337, 2000.

[20] P. Soo-Chang, Y. Min-Hung, and T. Chien-Cheng, "Discrete fractional Fourier transform based on orthogonal projections," Signal Processing, IEEE Transactions on, vol. 47, pp. 1335-1348, 1999. 


\section{Figures list}

\begin{tabular}{|c|c|}
\hline $\begin{array}{c}\text { Figure } \\
\text { No. }\end{array}$ & Figure captions \\
\hline 1 & Two targets scenario for Monopulse radar \\
\hline 2 & Basic structure of a monopulse radar \\
\hline 3 & Signal separation in the $a^{\text {th }}$ domain \\
\hline 4 & New structure of the proposed monopulse radar \\
\hline 5 & filtering radar data in FrFT domain \\
\hline 6 & $\begin{array}{l}\text { Conventional processor } \\
\text { (a) sum processor. } \\
\text { (b) difference processor. } \\
\text { (c) error voltage curve. } \\
\text { (d) processor output. }\end{array}$ \\
\hline 7 & $\begin{array}{l}\text { Spatial adaptive processor } \\
\text { (a) sum processor. } \\
\text { (b) difference processor. } \\
\text { (c) error voltage curve. } \\
\text { (d) processor output. }\end{array}$ \\
\hline 8 & $\begin{array}{l}\text { Conventional processor } \\
\text { (a) error voltage curve. } \\
\text { (b) processor output (No Filtering). } \\
\text { (c) processor output (FrFT Filtering). } \\
\text { (d) STDAE. }\end{array}$ \\
\hline 9 & $\begin{array}{l}\text { Spatial adaptive processor } \\
\text { (a) error voltage curve. } \\
\text { (b) processor output (No Filtering). } \\
\text { (c) processor output (FrFT Filtering). } \\
\text { (d) STDAE }\end{array}$ \\
\hline
\end{tabular}




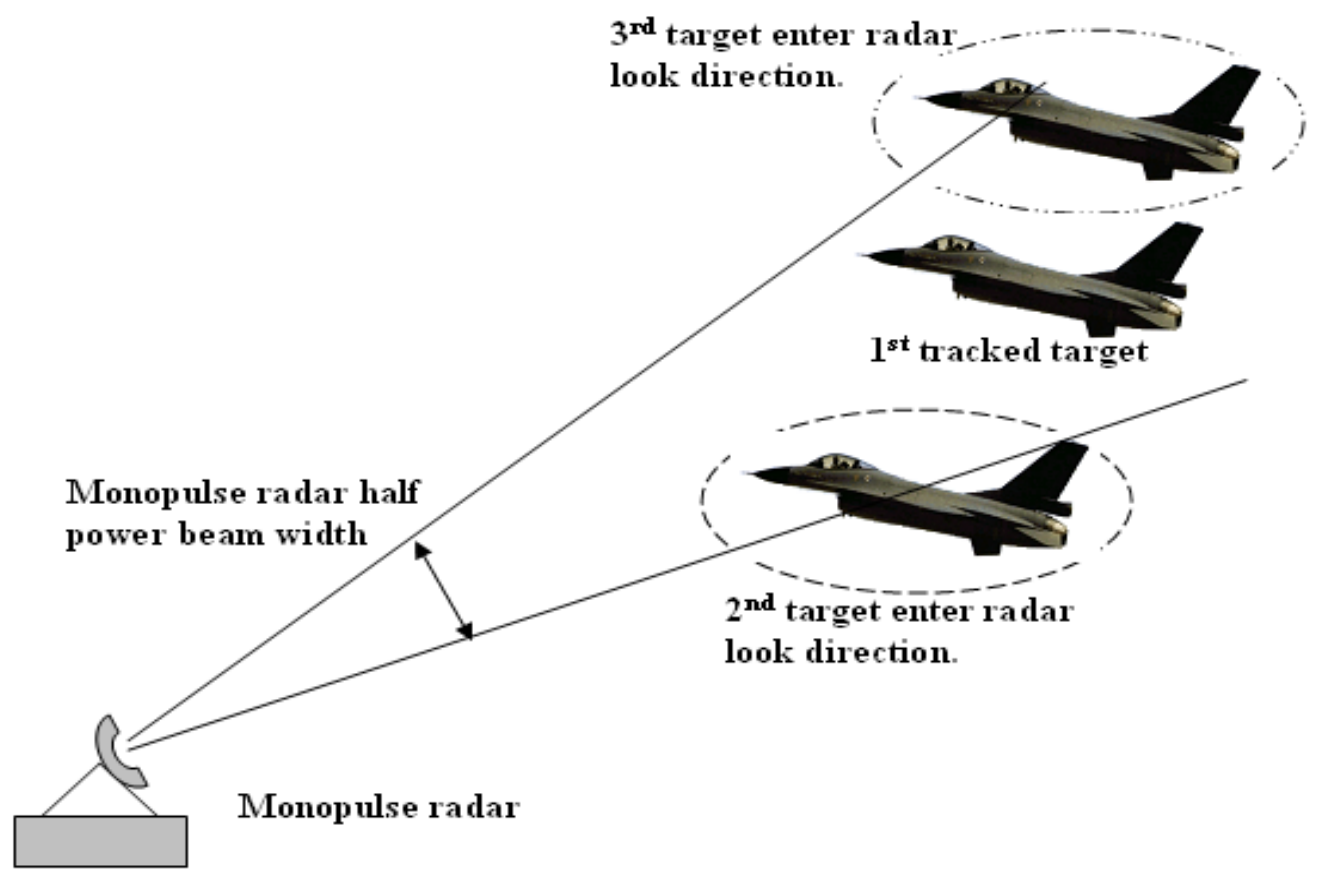

Fig 1- Two targets scenario for Monopulse radar

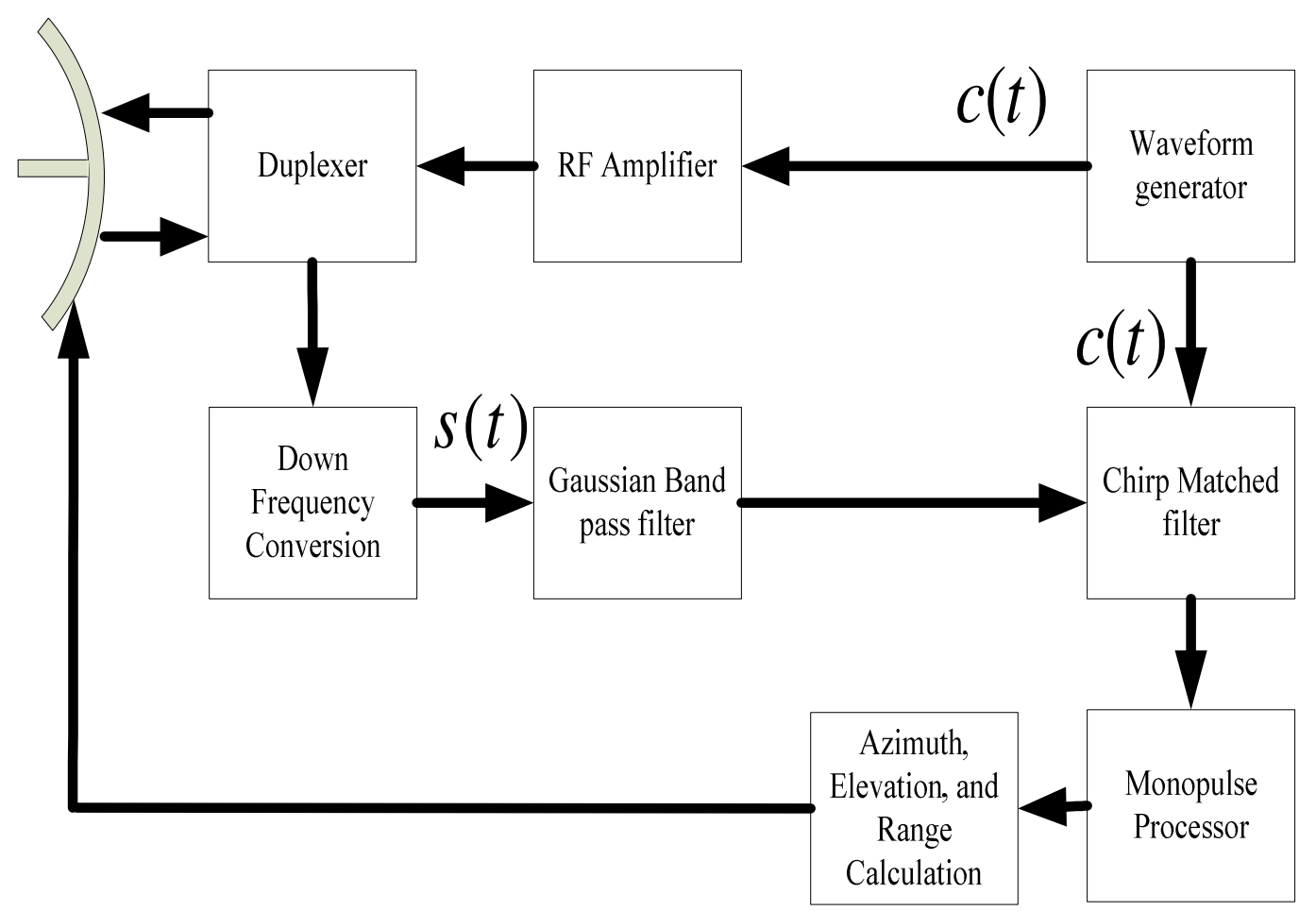

Fig 2-Basic structure of a monopulse radar 


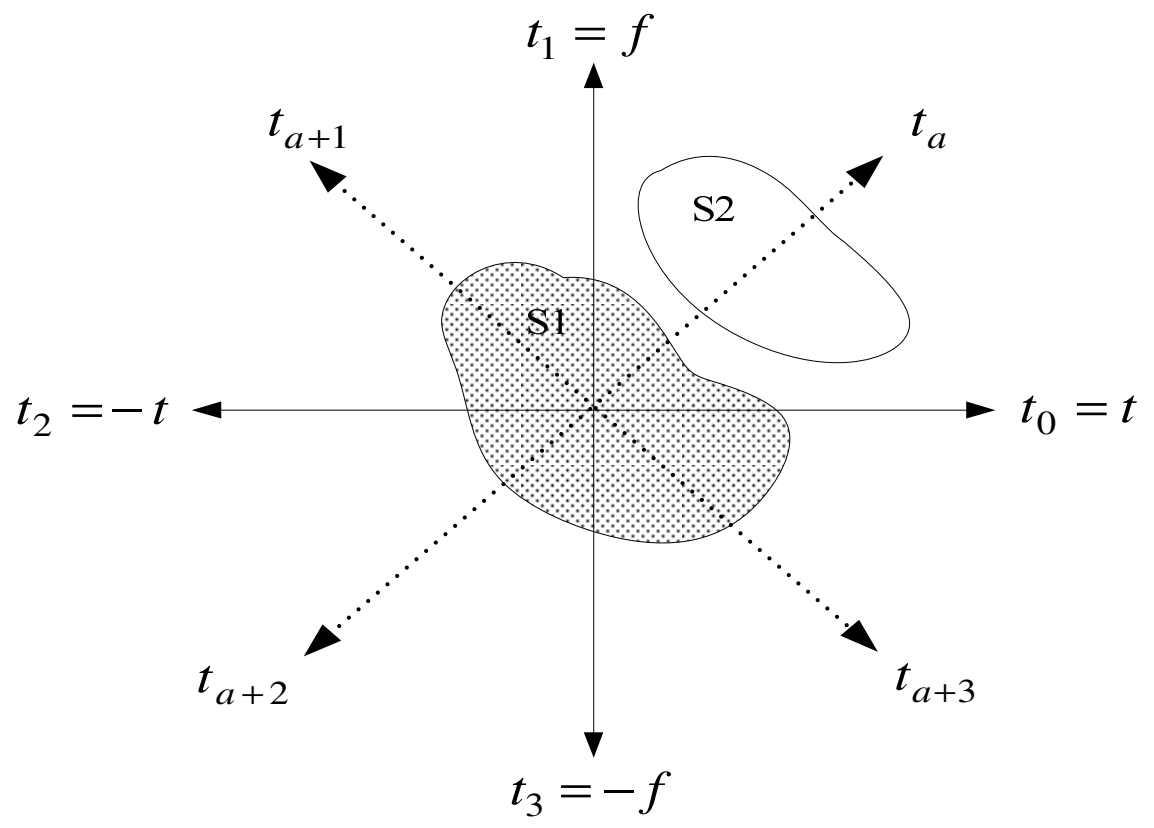

Fig 3- Signal separation in the $a^{\text {th }}$ domain

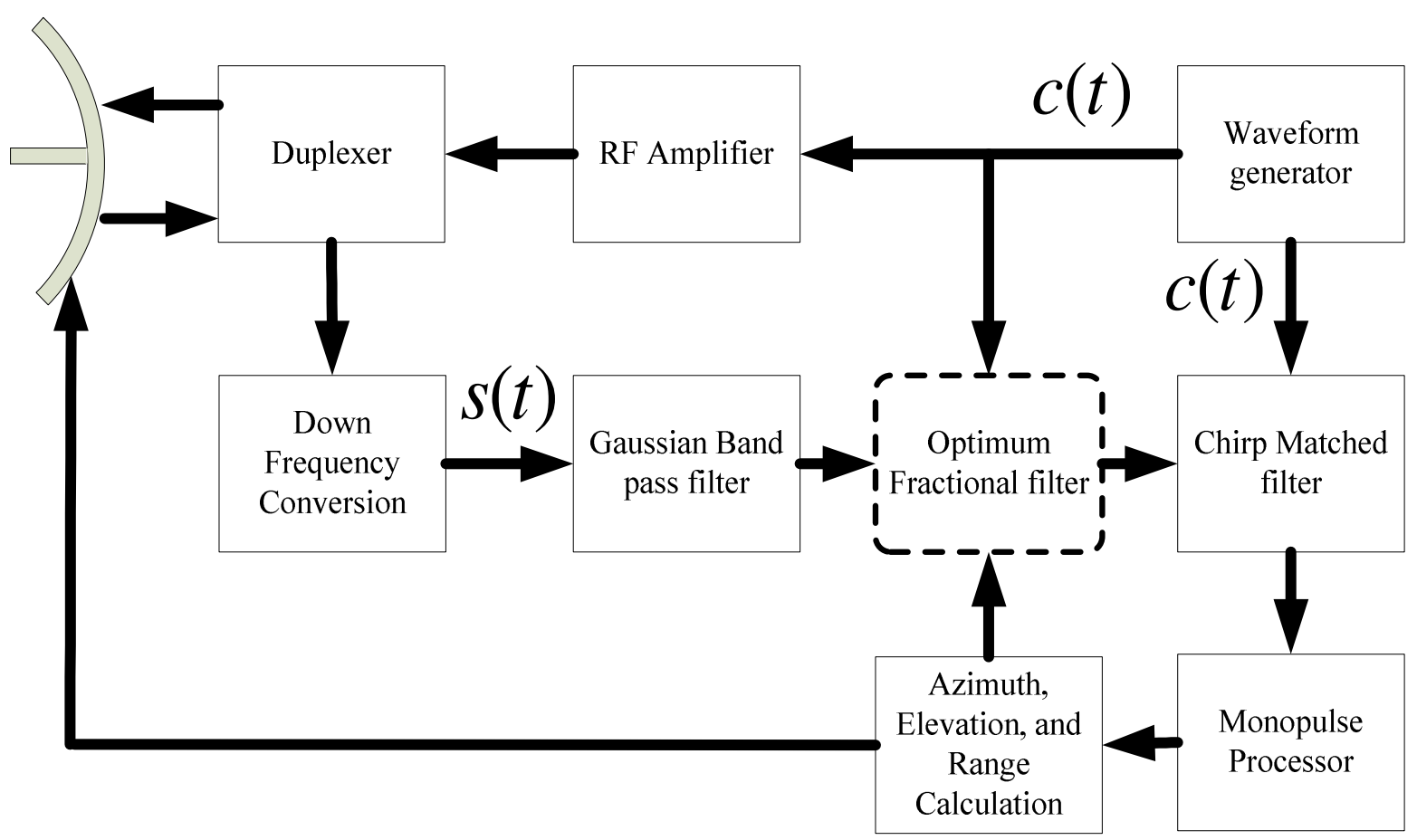

Fig 4- New structure of the proposed monopulse radar 


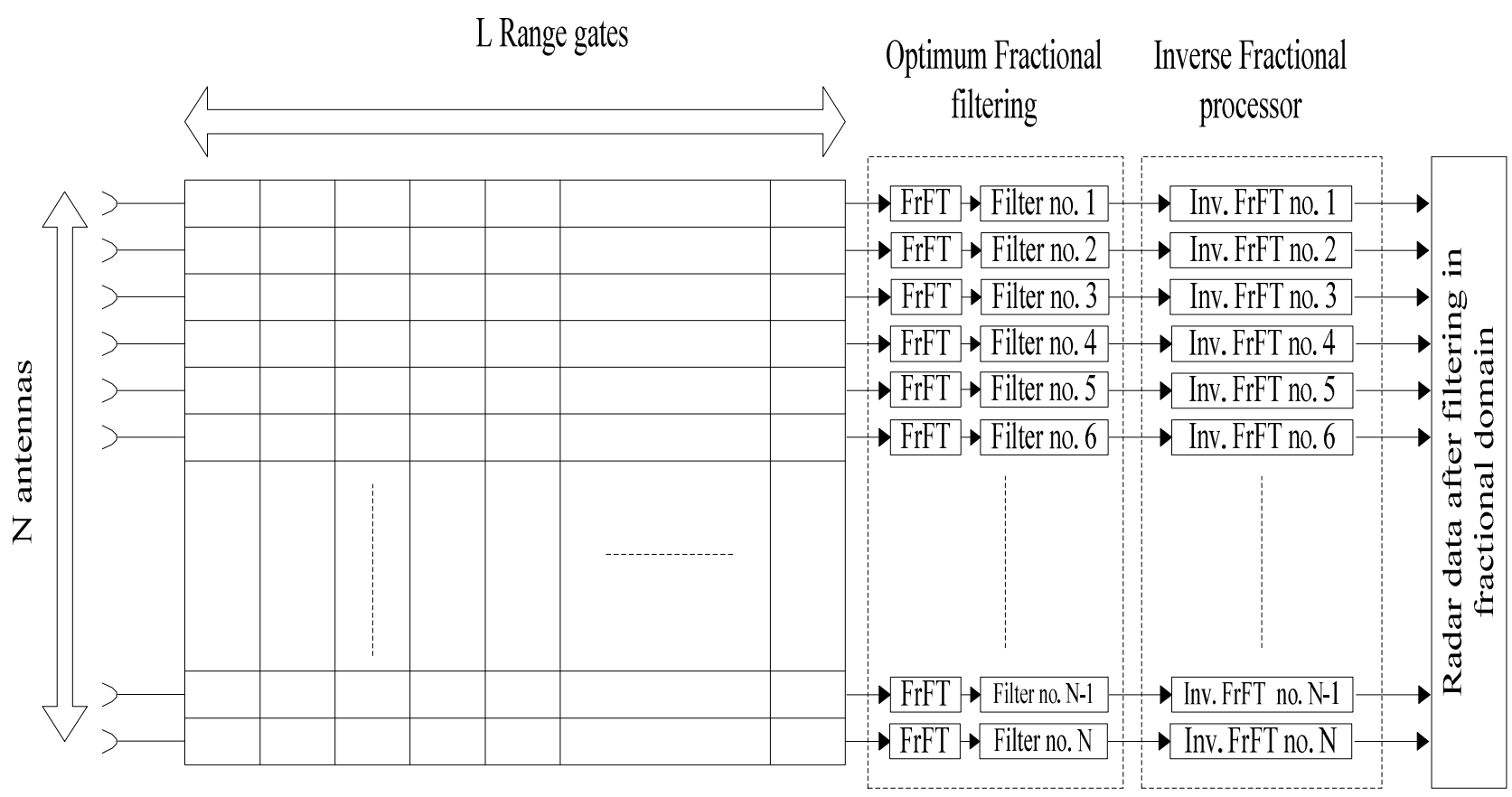

One pulse repetition interval

Fig 5- filtering radar data in FrFT domain 


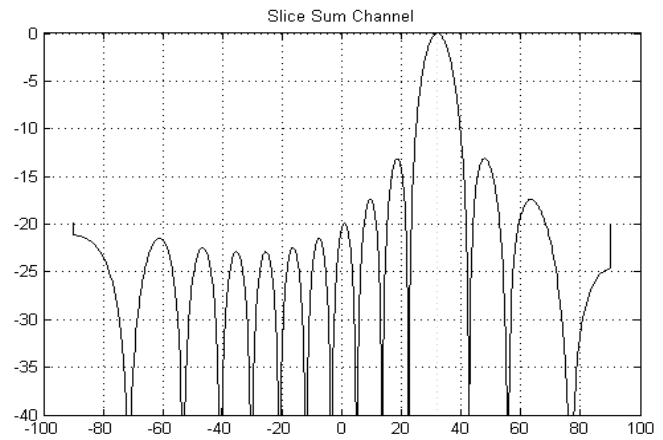

(a)

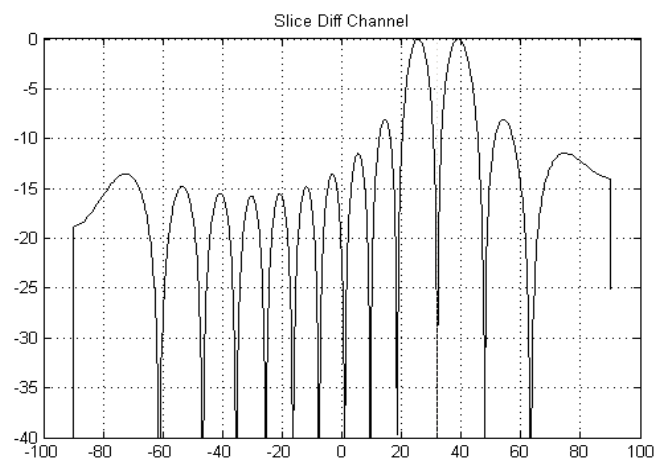

(b)

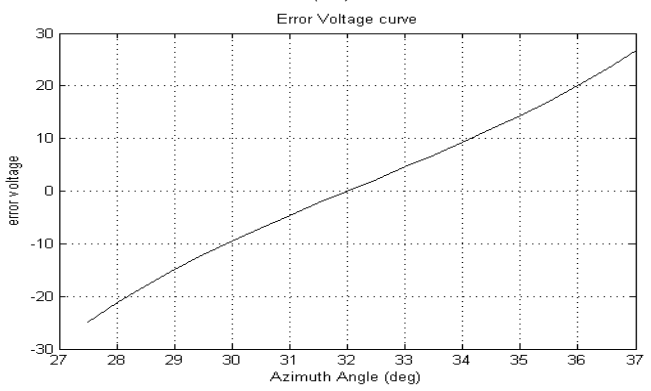

(c)

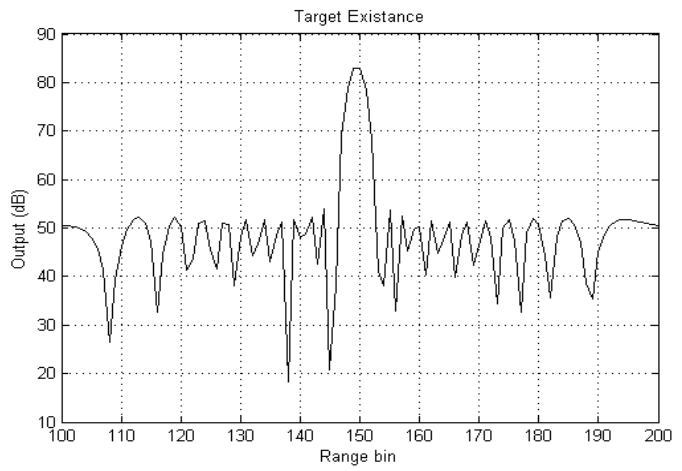

(d)

Fig 6-Conventional processor

(a) sum processor.

(b) difference processor.

(c) error voltage curve.

(d) processor output.

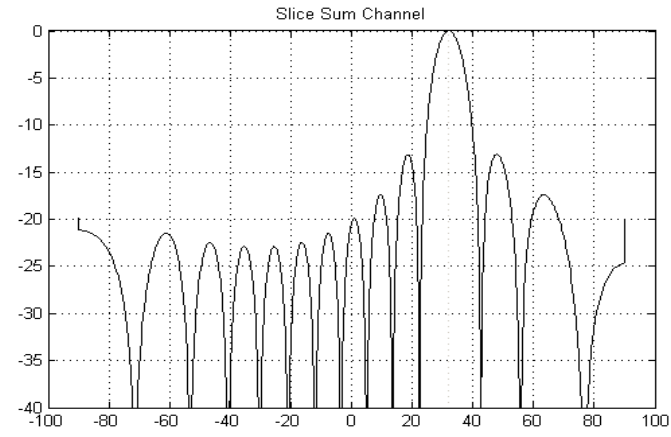

(a)

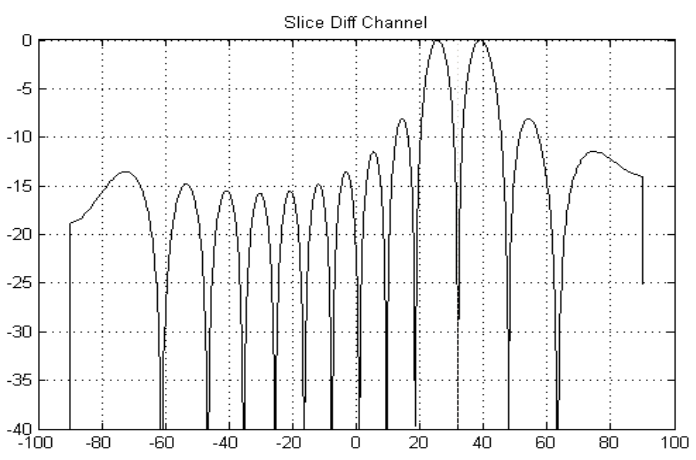

(b)

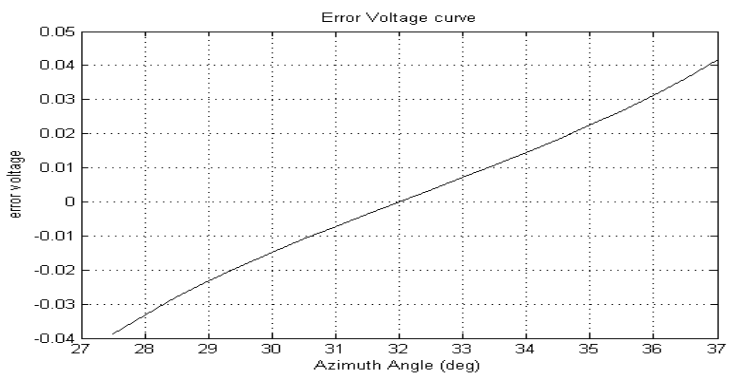

(c)

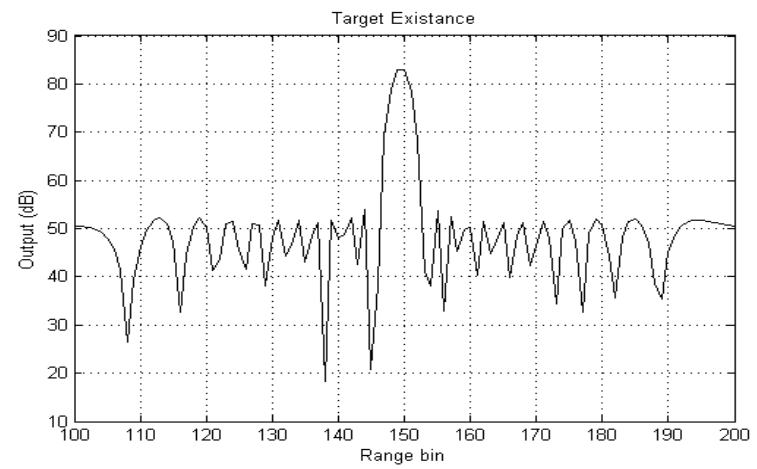

(d)

Fig 7- Spatial adaptive processor

(a) sum processor.

(b) difference processor.

(c) error voltage curve.

(d) processor output. 


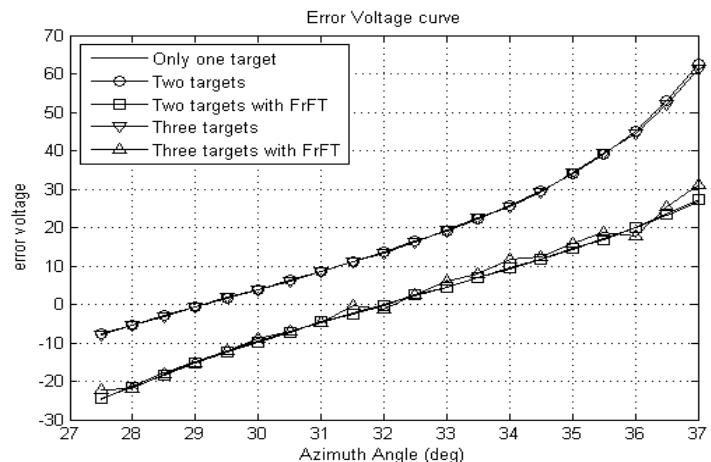

(a)

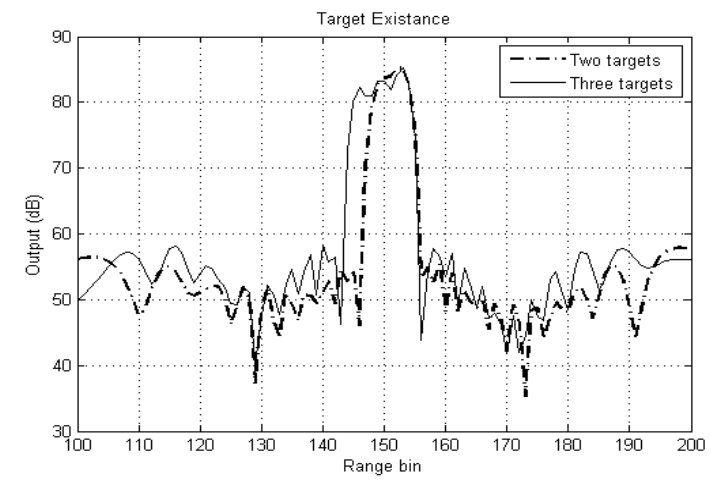

(b)

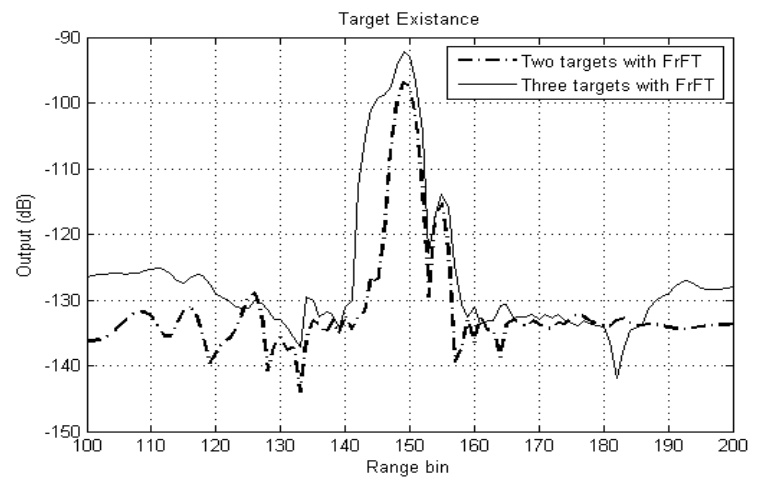

(c)

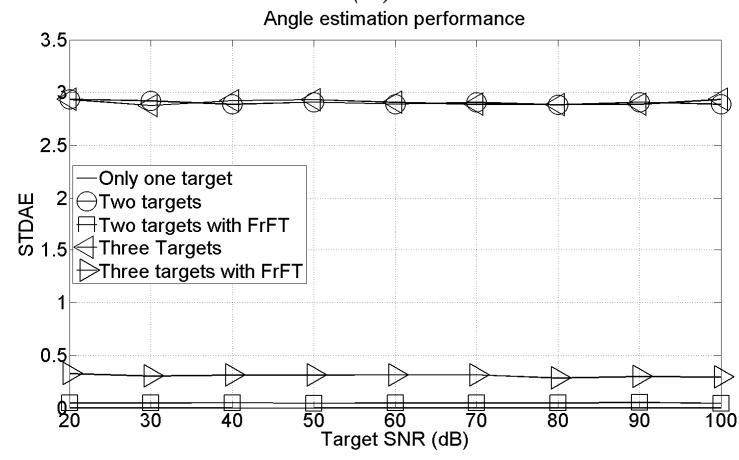

(d)

Fig 8-Conventional

(a) error voltage curve.

(b) processor output (No Filtering).

(c) processor output (FrFT Filtering).

(d) STDAE.

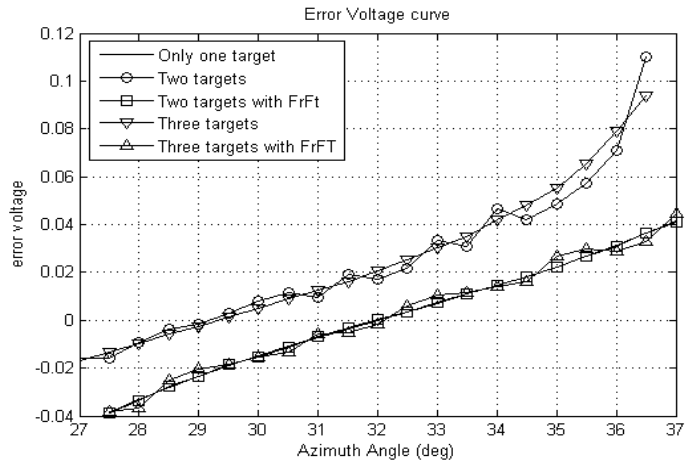

(a)

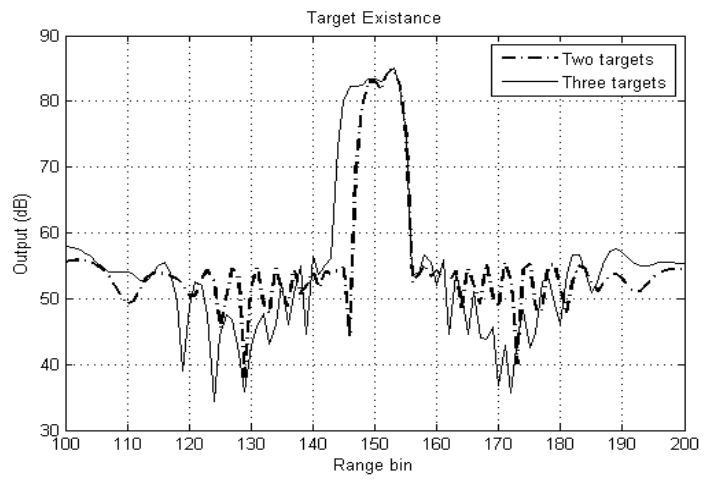

(b)

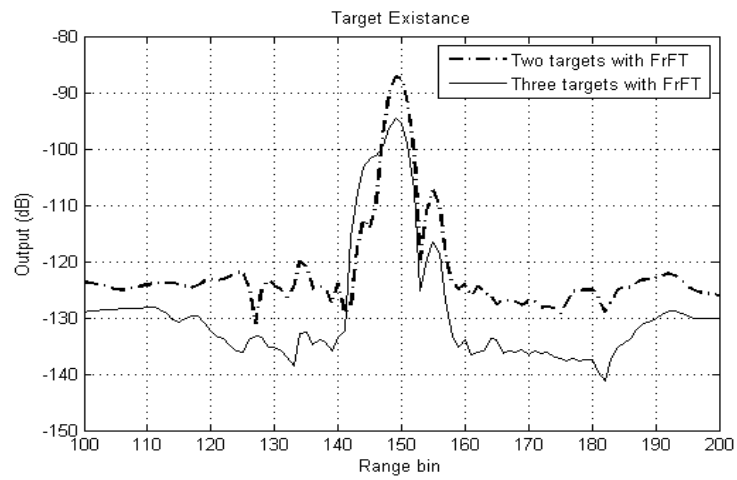

(c)

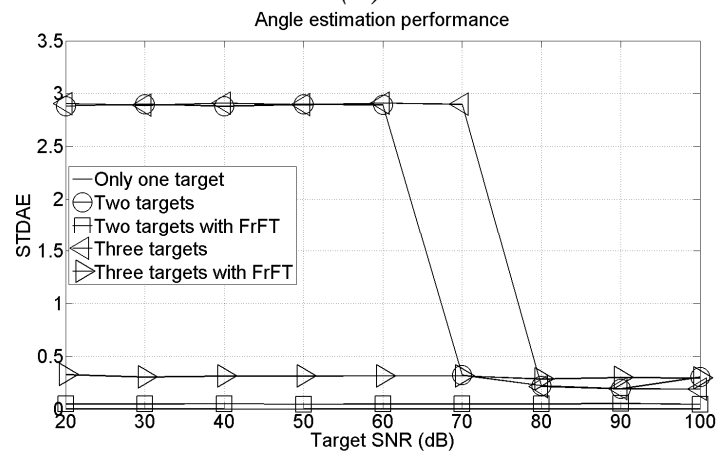

(d)

Fig 9- Spatial adaptive

(a) error voltage curve.

(b) processor output (No Filtering).

(c) processor output (FrFT Filtering).

(d) STDAE. 
